\title{
An Ultrastructural Study on the Retina of the Blind Cave Fish (Astyanax hubbusi)
}

\author{
David T. Yew and Henry M. Yoshihara \\ Department of Biology, The Chinese University \\ of Hong Kong, Hong Kong \\ and \\ Department of Anatomy, Wayne State University, \\ Detroit, Mich. 48201, U.S.A.
}

Received September 9,1975

The peculiar eyes of the blind cave fishes of the genera Anoptichthys and Astyanax have been studied by various authors (Gresser and Breder 1940, Breder and Gresser 1941a,b, Breder 1942, Lüling 1953, Peters and Peters 1966, Egar 1974, Sliger 1974). The eyes of Anoptichthys show various abnormal phenomena (Gresser and Breder 1940, Breder 1942, Lüling 1953). The eyes of the Astyanax, although structurally functional (Egar 1974), have been reported by Peters and Peters (1966) to display morphological variations.

An important question regarding the structure of the eyes of these forms relates to the structural integrity of those layers of the retina closely allied to the initiation and transmission of visual impulses. Thus, a study of the fine structure of the retinal layers of Astyanax hubbsi was undertaken.

\section{Materials and methods}

Specimens of Astyanax hubbsi were purchased from commercial sources. Eight eyes were dissected out and prefixed in phosphate buffered (pH 7.2) glutaraldehyde, then postfixed with buffered $1 \%$ osmium tetroxide. The specimens were dehydrated through a graded series of alcohols; cleared in propylene oxide and embedded in Epon. Thin sections were cut with a Porter-Blum ultramicrotome and stained with uranyl acetate and lead citrate then observed with a Zeiss E.M. $9 \mathrm{~S}_{2}$ electron microscope.

\section{Results}

Pigment epithelium: This layer lies immediately below Bruch's membrane; a structure consisting of a thick predominately collagenous matrix $0.3 \mu$ thick bordering a thin layer of basement membrane which serves as the basal lamina for the pigment epithelium. The pigment epithelium is composed of simple cuboidal cells which may be elongated in some specimens. In order to facilitate description, this layer can be divided into three zones, namely the basal zone nearest to the Bruch's membrane, the nuclear zone surrounding the nucleus and the apical zone nearest to the outer segments of the photoreceptors. 
In the basal zone, basal infoldings are not observed. Dense bodies, mitochondria and sparse canalicular rough endoplasmic reticulum are present. Large elongated unclei with distinct nuclear envelopes occupy the nuclear zone surrounded by pigment granules of varying densities and may be in different stages of develop-
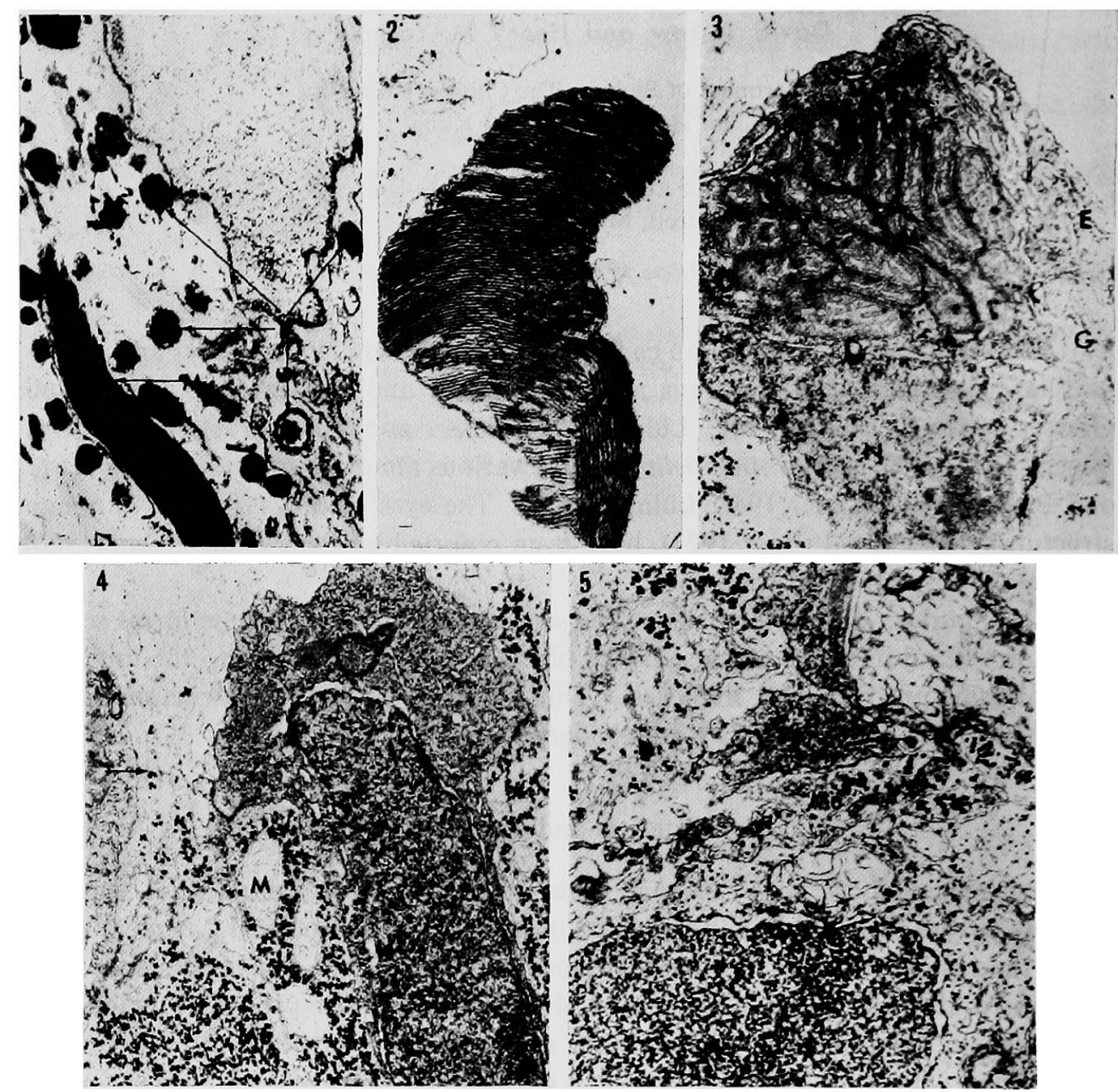

Fig. 1-5. 1, pigment epithelium of the blind cave fish showing My (Myeloid body) and pigment granules $(P) . \quad \times 33250$. 2, outer segment of photoreceptor of blind cave fish with disoriented lamellae (arrow). $\times 33250.3$, the bud like inner segment of photoreceptor of the blind cave fish. Note presence of mitochondria (M), rough endoplasmic reticulum (E) and golgi apparatus (G). Also note nucleus beneath the inner segment. $\times 33250$. 4, Muller cell $(\mathrm{Mu})$ intervenes between the photoreceptors. This cell has mitochondria (M) and microvilli projection (arrow) with glycogen. $\times 33250$. 5, outer plexiform layer of the cave fish retina. Note presence of synaptic ribbon (arrow) inside photoreceptor terminal (Pe). Also note nearby Muller (Mu). $\times 33250$.

ment. Rough endoplasmic reticulum and occasional dense bodies are also observed in this region. The apical zone, on the other hand, is typified by an increase in the number of pigment granules, parallel aligned rough endoplasmic reticulum, golgi apparatus and presence of distinct myeloid bodies (Fig. 1). Microvillous projections 
containing pigment granules and neurotubules are found originating from this regions.

Photoreceptors: The photoreceptors of blind cave fish contain essentially the same components as those of other vertebrates. The distal portions adjacent to the
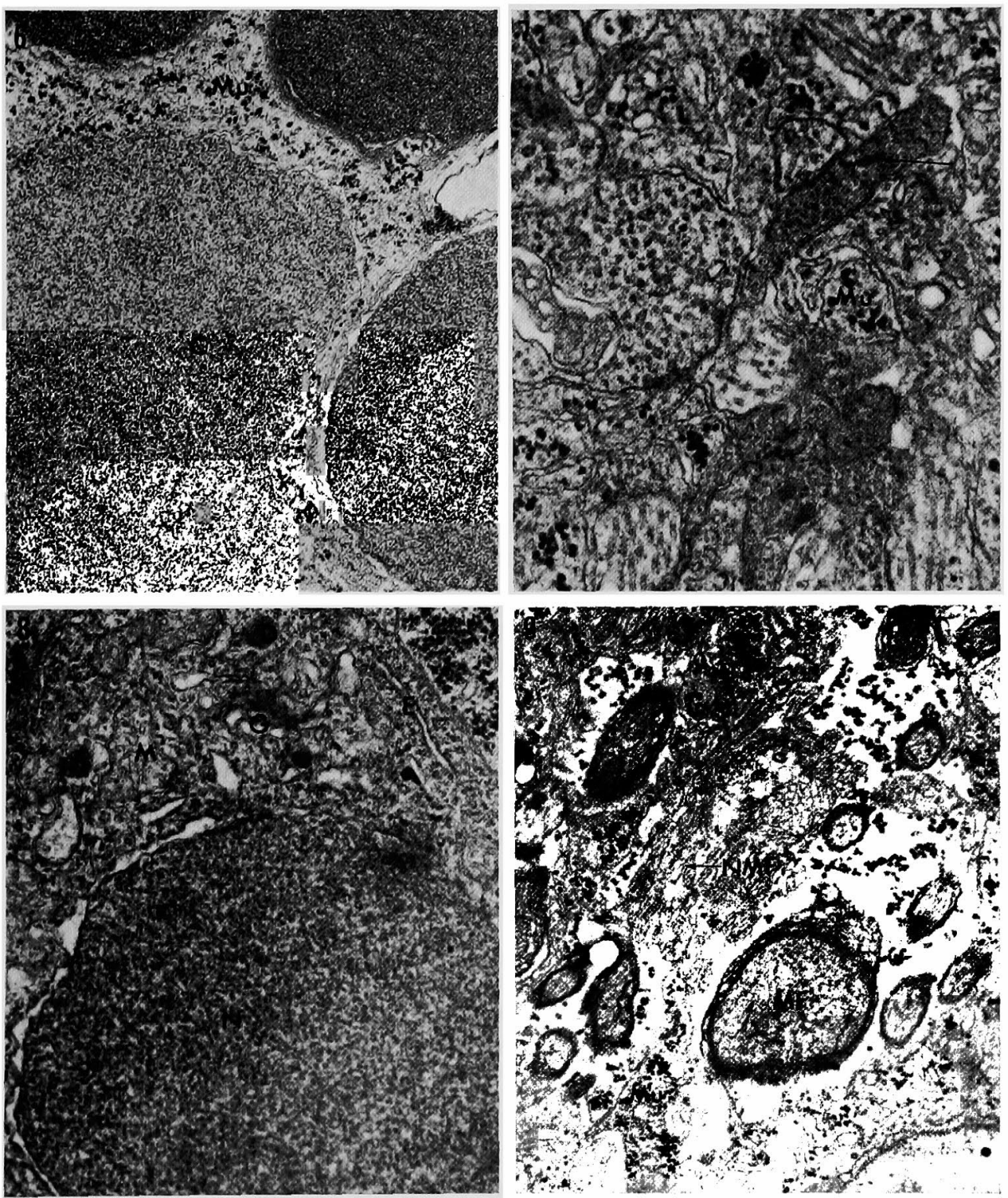

Figs. 6-9. 6, inner nuclear layer of the blind cave fish retina showing Muller (Mu) surrounding cell body $(\mathrm{C})$ in the inner nuclear layer. $\times 33250$. 7, inner plexiform layer of the blind cave fish retina. Note presence of synaptic ribbon (arrow) in this layer. $\times 33250.8$, ganglion cell of the cave fish retina with a large nucleus $(N)$, rough endoplasmic reticulum (E), golgi apparatus $(G)$, mitochondria (M) and dense body (D). $\times 33250$. 9, nerve fiber layer of the cave fish retina. Note presence of Myelinated fiber (MF), non Myelinated fiber (NMF) and branch of Muller cell $(\mathrm{Mu}) . \quad \times 33250$. 
pigment epithelium are outer segments which are stacks of flatten membranes. The proximal part are more cellular and constitute the inner segments. The outer segments are absent in some photoreceptors whereas in others, they are present but exhibiting various degrees of disorientation of lamellae (Fig. 2). The inner segments, on the other hand, are mostly bud-like protrusions containing darkly stained mitochondria and golgi apparatus (Fig. 3). These photoreceptors are separated from each other by Müller fibers possessing large amount of glycogen particles and a few lightly stained mitochondria (Fig. 4). At their apical regions microvilli measuring 0.3 to $0.5 \mu$ in length containing glycogen project into the optic ventricle. The nuclei of the photoreceptors (the outer nuclear layer) are arranged into several rows, the upper ones are more elongated in shape and the innermost ones are more conical.

Outer plexiform layer: This layer represents the neuronal connection between the photoreceptors and inner layers. Photoreceptor terminals are observed with an occasional synaptic ribbon (Fig. 5), a few synaptic vesicles and dense conventional junctions. Numerous branches of the Müller fibers loaded with glycogen particles invade between these terminals.

The inner nuclear layer: The cell bodies of the horizontal, bipolar and amarcine cells are present in this layer. These cells are lined by a scanty amount of cytoplasm containing golgi apparatus, mitochondria, rough endoplasmic reticulum and ribosomes in different proportions. They are again intervened by Müller branches loaded with glycogen (Fig. 6).

Inner plexiform layer: This layer represents neuronal connecting between the inner nuclear layer and the ganglion cells. Synaptic ribbons and dense junctions are all observed in this layer (Fig. 7). Müller branches with glycogen particles fill the spaces between the various fibers.

Ganglion cell layer and nerve fiber layer: Ganglion cells with dense bodies, rough endoplasmic reticulum, mitochondria (Fig. 8) and myelinated and nonmyelinated nerve fibers (Fig. 9) are enclosed by Müller branches with large amount of glycogen. These particles sometimes accumulate to form large body like forms.

\section{Discussion}

Fish in general do not have extensive basal infoldings in their pigment epithelium (Okuda 1962). This is true also for the blind cave fish. In this form, myeloid bodies are present. Myeloid bodies are thought to be intracellular photoreceptors (Porter and Yamada 1960), or light sensitive crystals (Yamada 1961) suggesting the neuroretina of this form retains the capacity to detect light.

Disorientations and absence of outer segments, presence of bud like inner segments, indicate on the other hand, these may be a deficiency in visual reception in this fish. However, in the plexiform layers synaptic ribbons and vesicles essential for impulse transmission are present (Dowling 1970), although in varying frequency and amount.

Furthermore, the large amount of glycogen particles in the Müller branches investing in between the different neuronal cells appear to point out that the retina 
requires a lot of storage for active metabolic processes and possibly as glycogen particles in the Müller. Glycogen presence in the Müller fibers have been reported in the mammals (Shimizu and Maeda 1953, Nakayama 1957, Raviola and Raviola 1962, Kawabara and Cogan 1963, Agarwal et al. 1964, Magalhaes and Coimbra 1970) and in birds (Uga and Smelser 1973).

The retinas of numerous cave species have been studied and recorded in the literature (Walls 1942), and many of which have shown some degrees of degeneration. The blind cave fish is no exception. Disoriented outer segments and short inner segments are features of their photoreceptors. However, apart from these deviations, the retina on a whole appears to resemble the functional retinas of many other vertebrates. It is therefore highly probably that the retina has the property to initiate and conduct impulse as suggested by some investigators (Egar 1974).

\section{Summary}

The retinal layers of the blind cave fish, Astyanax hubbsi were studied by electron microscopy. In the pigment epighelium, myeloid bodies are present. Disoriented outer segments and short inner segments are observed. A few synaptic ribbons and vesicles are located in the plexiform layers. Large amount of glycogen particles are seen in the Müller fiber surrounding the neuronal cells.

\section{Acknowledgement}

The authors are grateful to Dr. David B. Meyer for his generosity in allowing us to use his Electron microscope and facilities. We wish to thank Mrs. Amy Shea for typing the manuscript. One of the author (D. T. Yew) wants to dedicate this paper to his wife for appreciation of her continuous struggle with him through the past years.

\section{Literature}

Agarwal, L. P., Lamba, P. A., Mohan, M. and Batta, R. K. 1964. Histochemical study of retinal glycogen I. Normal and starvation. Orient. Arch. Ophthal. 2: 191-199.

Breder, C. M. Jr. 1942. Descriptive ecology of la Cueva Chica with especial reference to the blind fish Anoptichthys. Zoologica 27: 7-16.

- and Gresser, E. B. 1941a. Correlations between structural eye defects and behavior in the Mexican blind characin. Zoologica 26: 123-131.

- and - 1941b. Further studies on the light sensitivity and behavior of the Mexican blind characin. Zoologica 26: 289-296.

Dowling, J. E. 1970. Organization of vertebrate retinas. Invest. Opthal. 9: 655-680.

Egar, M. W. 1974. An ultrastructural study of the optic nerve of the blind cave fish. Anat. Rec.: 178-350.

Gresser, E. B. and Breder, Jr. C. M. 1940. The histology of the eye of the cave characin, Anoptichthys. Zoologica 25: 113-116.

Kuwabara, T., and Cogan, D. G. 1963 . Glycogen in the retina. Ann. Histochim. 8: 223-228.

Lüling, K. H. 1953. Über die fortschreitende Augendegenerations des Anoptichthys jordani Hubbs und Innes (Characidae). Zool. Anz. 151: 289-299. 
Magalhaes, M. M., and Coimbra, A. 1970. Electron microscope radioautographic study of glycogen synthesis in rabbit retina. J. Cell. Biol. 47: 263-275.

Nakayama, K. 1957. Histochemical study on the human fetal retina in the course of development. J. Clin. Ophthal. Tokyo 11: 1024-1032.

Okuda, K. 1962. Electron microscopic observations of the retinal pigment epithelium of vertebrate animals. Jap. J. Ophth. 6: 76-87.

Peters, N. and Peters, G. 1966. Das Auge zweier Hohlenformen von Astyanax mexicanus (Philippi) (Characinicae, Pisces). Roux' Arch. für Entwicklungsmechanik. 157: $393-$ 414.

Porter, K. R. and Yamada, E. 1960. Studies on the endoplasmic reticulum V. Its form and differentiation in the pigment epithelial cells of the frog retina. J. Biophys. Biochem. Cytol. 8: 181-205.

Raviola, E. and Raviola, G. 1962. Ricerche istochemiche sulle retina diconiglio nel corso dello sviluppo postnatale. Z. Zellforsch. 56: 552-572.

Shimizu, N. and Maeda, S. 1953. Histochemical studies on glycogen of the retina. Anat. Rec. 116: 427-438.

Sliger, C. 1974. An investigation of tectal efferents in the blind cave fish, A. hubbsi. Anat. Rec.: $178-467$.

Uga, S. and Smelser, G. K. 1973. Electron microscopic study of the development of retinal Müller cells. Invest. Ophth. 12: 295-307.

Walls, G. E. 1967. The Vertebrate Eye and its Adaptive Radiation. Hafner Co., N.Y.

Yamada, E. 1961. The fine structure of the pigment epithelium in the turtle eye. In "The Structure of the Eye", G. K. Smelser ed., Academic Press. N.Y.: 73-84. 